\title{
Calidad de vida en niños y adolescentes con trastorno del espectro autista sin discapacidad intelectual
}

\author{
José Luis Cuesta-Gómez, Ruth Vidriales-Fernández, Fernando Carvajal-Molina
}

Introducción. El concepto de calidad de vida tiene gran importancia en la intervención dirigida a las personas con discapacidad. Resulta indiscutible que el alcance de una calidad de vida satisfactoria debe ser el objetivo de las intervenciones clínicas dirigidas al colectivo de personas con trastorno del espectro autista (TEA), y un elemento clave para diseñar los tratamientos necesarios que mejoren el pronóstico de desarrollo futuro. A pesar de ello, el conocimiento sobre las condiciones objetivas y subjetivas que predicen una mayor satisfacción en las personas con TEA es aún muy limitado.

Objetivo. Explorar la percepción de la calidad de vida que experimenta un grupo de niños y adolescentes con TEA sin discapacidad intelectual asociada.

Pacientes y métodos. Muestra de 12 niños y 11 adolescentes con TEA sin discapacidad intelectual asociada. Dependiendo de la edad, se les aplicó el cuestionario de calidad de vida específico.

Resultados. Ambos grupos informan positivamente sobre su calidad, sin que existan diferencias en las dimensiones básicas. No obstante, en el caso de los adolescentes se obtienen puntuaciones más bajas en el área de autodeterminación en comparación con las demás.

Conclusiones. La discusión se centra en la identificación de las dimensiones más relevantes para la calidad de vida, las implicaciones para la intervención y la necesidad de adecuar las metodologías empleadas para la evaluación subjetiva de estos factores, debido a que las características de los TEA pueden alterar las habilidades necesarias para la valoración de la experiencia personal y requerir la adaptación de los procedimientos empleados.

Palabras clave. Autismo. Autoevaluación. Calidad de vida. Evaluación cualitativa. Satisfacción personal. Síndrome de Asperger. Trastorno del espectro autista.

\section{Introducción}

La atención al autismo en la literatura científica se ha incrementado significativamente en la última década y se han producido relevantes avances en su conocimiento y conceptualización. Uno de los cambios principales ha sido la consolidación del término 'trastorno del espectro autista' (TEA) [1], que enfatiza la enorme heterogeneidad de la presentación clínica de los TEA y de las necesidades de las personas que los presentan, así como la específica alteración en el desarrollo sociocomunicativo que comparten.

Estos avances han sido también reconocidos en la quinta edición del Manual diagnóstico y estadístico de los trastornos mentales (DSM-5) [2], que incorpora la categoría 'trastorno del espectro del autismo' enmarcada en una nueva sección de 'trastornos del neurodesarrollo'

El DSM-5 ha modificado también los criterios para el diagnóstico de TEA, unificando las áreas de comunicación y competencia social, y ampliando la exigencia en el número de síntomas necesarios en esta dimensión con el objetivo de incrementar la especificidad de los diagnósticos. Además, se han revisado y matizado algunos criterios establecidos en el área de los intereses restringidos, incluyendo las alteraciones en el procesamiento de información sensorial que se presentan asociadas con frecuencia a los TEA, como un nuevo criterio diagnóstico [1].

Otra de las aportaciones del DSM-5 es la incorporación de indicadores sobre la gravedad y severidad de la sintomatología, que permiten describir e identificar las necesidades de apoyo que la persona precisa en las dos principales áreas que definen los TEA, diferenciando tres niveles distintos: 'necesita ayuda muy notable' (grado 3), 'necesita ayuda notable' (grado 2) y 'necesita ayuda' (grado 1).

Aunque, como afirman Artigas y Paula [3], los nuevos criterios establecidos en el DSM-5 no han estado exentos de crítica y polémica, permiten armonizar en una categoría diagnóstica amplia las características nucleares de los TEA, con la presencia o no de dificultades cognitivas o de lenguaje, así co-
Departamento de Ciencias de la Educación; Facultad de Educación; Universidad de Burgos; Burgos (J.L. Cuesta-Gómez). Asesoría Técnica; Confederación Autismo España (R. Vidriales-Fernández). Departamento de Psicología Biológica y de la Salud; Facultad de Psicología; Universidad Autónoma de Madrid; Madrid, España (F. Carvajal Molina).

Correspondencia:

Dr. José Luis Cuesta Gómez. Facultad de Educación. Universidad de Burgos. Villadiego, s/n. E-09001 Burgos.

E-mail:

jlcgomez@ubu.es

Declaración de intereses: Los autores manifiestan la inexistencia de conflictos de interés en relación con este artículo.

Aceptado tras revisión externa: 27.01.16.

Cómo citar este artículo: Cuesta-Gómez JL, VidrialesFernández R, Carvajal-Molina F. Calidad de vida en niños y adolescentes con trastorno del espectro autista sin discapacidad intelectual. Rev Neurol 2016; 62 (Supl 1): S33-9.

(c) 2016 Revista de Neurología 
mo la presencia o no de enfermedad genética o médica o factor ambiental conocido [4].

Los trabajos y publicaciones que exploran la percepción de las personas con TEA sin discapacidad intelectual asociada sobre su condición diagnóstica y su experiencia cotidiana ponen de manifiesto la enorme sensación de 'no encajar' en la sociedad y el malestar personal que sufren, evidenciando estados de ansiedad, depresión, etc., con mayor frecuencia que las personas con TEA con discapacidad intelectual [5-7]. Estas percepciones se relacionan tanto con las propias manifestaciones clínicas del trastorno, como con su relativa 'invisibilidad' y falta de comprensión por parte de la sociedad. La apariencia de 'normalidad' y la sutileza de los déficits (difíciles de identificar) característicos de los TEA sin discapacidad intelectual asociada fomentan una visión negativa, excéntrica y rara de la persona que lo presenta, pero sin que los demás lleguen a comprender la naturaleza y el impacto de la discapacidad social que implica [8].

Por ello, la intervención y los apoyos deben plantearse necesariamente desde una perspectiva integral, considerando tanto las propias características del individuo como sus expectativas y los contextos en los que se va a desenvolver. Teniendo en cuenta lo anterior, en el caso de las personas con TEA sin discapacidad intelectual, las necesidades se derivan principalmente de las elevadas exigencias sociales de la vida cotidiana, de las propias alteraciones en las capacidades cognitivas necesarias para responder a ellas, y también de la falta de ajuste entre estos aspectos y las expectativas que los demás tienen sobre la persona [9].

Es en este ámbito donde cobra una especial relevancia el paradigma de calidad de vida como marco general para organizar la intervención. El modelo adoptado en este trabajo es el desarrollado por Schalock y Verdugo [10-12], que define la calidad de vida como un estado deseado de bienestar personal, de carácter multidimensional, que tiene propiedades universales y propiedades ligadas a la cultura, y que está influenciado por las características personales y por los factores ambientales. Este modelo se concreta a través de dimensiones, indicadores y resultados personales [13].

Son muy pocas las investigaciones realizadas específicamente sobre la calidad de vida de las personas con TEA sin discapacidad intelectual, aunque en los últimos años se detecta un incremento del interés por este conocimiento [14-18]. También se han desarrollado investigaciones específicas sobre calidad de vida en personas con TEA y discapacidad intelectual asociada [19-26].
La mayoría de las investigaciones se han realizado empleando medidas cualitativas sobre la calidad de vida percibida por adultos, y comparando los resultados en ocasiones con grupos de adultos sin alteraciones en el desarrollo [9]. En general, los resultados señalan que la valoración global que los adultos con TEA hacen sobre su calidad de vida es pobre, y que no se relaciona directamente con el nivel cognitivo de los participantes ni con la gravedad de sus manifestaciones clínicas $[9,16]$.

Además, ponen de manifiesto que la valoración depende en mayor medida de la discrepancia que las personas con TEA perciben entre los apoyos que reciben y los que consideran que necesitan, más que de los recursos o apoyos que disfrutan en realidad. Estos trabajos concluyen que es imprescindible incluir la valoración que la persona con TEA hace de su situación y de sus necesidades en cualquier intervención o tratamiento que reciba.

Los trabajos que exploran las dimensiones con mayor influencia en la calidad de vida de los niños y adolescentes con TEA sin discapacidad intelectual asociada son muy escasos. Sus conclusiones destacan, en el caso de las personas con TEA, que existen diferencias en la valoración de las relaciones interpersonales de amistad, y ponen de manifiesto que su calidad tendría un mayor valor predictivo de la satisfacción que la cantidad de relaciones disponibles, cuando se comparan con otras personas sin alteraciones en el desarrollo. Asimismo, ponen de manifiesto que los logros académicos en la niñez y la adolescencia no predicen la calidad de vida en la etapa adulta, manteniéndose una baja satisfacción laboral y dificultades significativas para el disfrute de una vida independiente, a pesar de haber alcanzado logros educativos significativos. Sin embargo, el disfrute de un empleo satisfactorio en la vida adulta sí se relaciona con la percepción de una calidad de vida satisfactoria [9].

Los resultados anteriores confirman la necesidad de incrementar el conocimiento sobre la calidad de vida percibida de los niños y adolescentes con TEA, de manera que desde la intervención clínica se puedan fomentar las habilidades y competencias necesarias para experimentar una satisfactoria calidad de vida en el futuro.

Por tanto, el objetivo general de este estudio es explorar de manera preliminar la evaluación subjetiva que los niños y adolescentes con TEA hacen de su calidad de vida. Asimismo, se pretende explorar si existen diferencias entre las dimensiones más relevantes para la calidad de vida subjetiva de los niños y los adolescentes con TEA asociadas a la edad. 


\section{Pacientes y métodos}

Se emplea un muestreo no aleatorio, a propósito, incluyendo a los participantes en función de criterios de expertos. Para la participación se requiere que la persona tenga entre 8 y 18 años y presente un diagnóstico confirmado de TEA sin discapacidad intelectual asociada -cociente intelectual total (CIT), valorado con pruebas estandarizadas, superior a 70- basándose en los criterios establecidos en el DSM-5 [2].

Además, se solicita que los participantes cuenten con valoraciones realizadas con la escala de observación para el diagnóstico del autismo (ADOS-G) [27], superando las puntuaciones de corte establecidas para los TEA.

El acceso y contacto con los participantes se ha establecido a través de servicios especializados que les prestan apoyo psicológico e intervención clínica.

En todos los casos, el protocolo de investigación y el consentimiento informado ha sido aprobado previamente por la organización a la que pertenecen las personas con TEA, y firmado por ellas mismas o por sus representantes legales.

Los participantes han sido 23, distribuidos en dos grupos, en función de su edad. El grupo de niños ha estado integrado por 12 participantes, 11 niños y una niña, con edades comprendidas entre 8 y 11 años.

El grupo de adolescentes ha estado formado por un total de 11 participantes, 10 chicos y una chica, con edades comprendidas entre 12 y 18 años. Los descriptivos referidos a la edad y el CIT de ambos grupos se recogen en la tabla I. Al hacer comparaciones mediante el estadístico $U$ de Mann-Whitney, como cabía esperar, la diferencia de edad entre ambos grupos resultó significativa $(p<0,001)$ y, en cambio no hubo diferencias referidas al CIT $(p=1)$.

Se ha establecido un diseño transversal empleando una metodología mixta de recogida de información, y combinando tanto técnicas cuantitativas (cuestionarios) como cualitativas (protocolo de entrevista elaborado ad hoc para el proyecto).

El cuestionario de calidad de vida en la infancia [28] y el cuestionario de evaluación de la calidad de vida de alumnos adolescentes [29] son los instrumentos cuantitativos elegidos para evaluar las percepciones subjetivas de los participantes sobre su calidad de vida.

El cuestionario de calidad de vida en la infancia explora cinco dominios de calidad de vida, es autoadministrable y resulta apropiado para niños de 8-11 años. Se compone de 53 ítems, con cuatro opciones de respuesta, está diseñado para reflejar la percep-
Tabla I. Edades y puntuaciones de cociente intelectual total (CIT) de los niños y adolescentes participantes.

\begin{tabular}{llc}
\hline & & Media \pm desviación estándar \\
\hline \multirow{2}{*}{ Niños } & Edad (años) & $9,00 \pm 1,78$ \\
\cline { 2 - 3 } & CIT & $99,33 \pm 19,84$ \\
\hline \multirow{2}{*}{ Adolescentes } & Edad (años) & $15,09 \pm 2,02$ \\
\cline { 2 - 3 } & CIT & $97,66 \pm 14,45$ \\
\hline
\end{tabular}

ción de bienestar desde la perspectiva infantil, y se contempla que pueda administrarse a niños con necesidades educativas especiales.

El cuestionario de evaluación de la calidad de vida de alumnos adolescentes está dirigido a niños y jóvenes de 12-18 años. Se estructura en dos partes, una que recoge información cuantitativa y otra cualitativa. La primera está compuesta por 61 ítems, en una escala de tipo Likert, que evalúan siete de las dimensiones de calidad de vida. La segunda parte está compuesta por 10 preguntas abiertas sobre la satisfacción de la persona con su vida en general y con las distintas dimensiones en particular.

Para recoger información cualitativa adicional sobre la percepción que todos los participantes tienen sobre su calidad de vida, se diseñó un protocolo de información específico. En él se solicita información sobre los aspectos que resultan más relevantes para la calidad de vida de la persona en todas las dimensiones del modelo de Schalock y Verdugo [10], así como sobre los elementos precisos que podrían ayudar a favorecerla.

El contenido y estructura del cuestionario fue contrastado por un grupo de cuatro expertos independientes, especializados en el diagnóstico y la intervención clínica para personas con TEA sin discapacidad intelectual. Éstos realizaron las modificaciones oportunas para que resultara accesible y conceptualmente comprensible a los participantes (estructuración de la información, reducción de lenguaje figurado u otros elementos de difícil interpretación, etc.).

La recogida de la información se realizó de manera individual, y en todos los casos se administró por un profesional especializado, vinculado a la intervención. Para hacerlo, el profesional debía haber intervenido durante al menos 12 meses con la persona a la que entrevistaba.

Se contempló la posibilidad de realizar la evaluación en más de una sesión si fuera necesario. 
Tabla II. Medias \pm desviaciones estándares en las dimensiones de calidad de vida: $\operatorname{niños}(n=12)$.

\begin{tabular}{lc}
\hline Bienestar emocional & $2,8846 \pm 0,58629$ \\
\hline Bienestar físico & $3,2361 \pm 0,39860$ \\
\hline Bienestar material & $3,1667 \pm 0,37605$ \\
\hline Desarrollo personal & $3,1136 \pm 0,34263$ \\
\hline Relaciones interpersonales & $3,0049 \pm 0,43477$ \\
\hline
\end{tabular}

Tabla III. Medias \pm desviaciones estándares en las dimensiones de calidad de vida: adolescentes $(n=11)$.

\begin{tabular}{ll}
\hline Bienestar emocional & $3,2864 \pm 0,42868$ \\
\hline Bienestar físico & $3,4182 \pm 0,44231$ \\
\hline Bienestar material & $2,8064 \pm 0,57342$ \\
\hline Desarrollo personal & $3,2991 \pm 0,47507$ \\
\hline Relaciones interpersonales & $3,1545 \pm 0,25442$ \\
\hline Integración en la comunidad & $2,9587 \pm 0,53065$ \\
\hline Autodeterminación & $2,6623 \pm 0,44388$ \\
\hline
\end{tabular}

\section{Resultados}

Los resultados obtenidos hacen referencia a los datos descriptivos generales sobre los participantes y a los análisis de los datos cuantitativos obtenidos en la evaluación subjetiva de su calidad de vida. En este estudio no se han incluido los análisis de la información cualitativa.

Dado el tamaño de la muestra y que el número de dimensiones valoradas por los cuestionarios es diferente, se decidió realizar un análisis independiente para cada grupo comparando los datos en las distintas áreas valoradas mediante la prueba de Friedman.

En el grupo de los niños (Tabla II), dicho análisis no resultó significativo $\left(\chi_{(4)}^{2}=3,15 ; p=0,53\right)$, y no se hallaron diferencias entre las cinco áreas que recoge el cuestionario (bienestar físico, bienestar material, bienestar emocional, desarrollo personal y relaciones interpersonales).

En cambio, en el grupo de los adolescentes ( $\mathrm{Ta}-$ bla III), el análisis sí resultó significativo $\left(\chi_{(6)}^{2}=21,87\right.$; $p<0,001)$ al comparar las siete áreas que recoge el cuestionario (bienestar físico, bienestar emocional, bienestar material, desarrollo personal, relaciones interpersonales, inclusión en la comunidad y autodeterminación). Las comparaciones posteriores indicaron que las puntuaciones más bajas de este grupo se obtuvieron en la dimensión de autodeterminación, diferencia que fue significativa en relación con todas las áreas $\left(p_{\mathrm{s}}<0,05\right)$ salvo con la de bienestar material. De hecho, aunque no alcanzó el nivel de significación, bienestar material puntuó más bajo que bienestar físico $(p=0,062)$, desarrollo personal $(p=0,059)$ y relaciones interpersonales $(p=0,056)$.

Asimismo, las puntaciones en integración en la comunidad resultan significativamente inferiores a las obtenidas en bienestar físico y desarrollo personal $\left(p_{\mathrm{s}}<0,05\right)$.

Para analizar las diferencias entre los grupos se realizaron comparaciones independientes para cada una de las cinco dimensiones comunes evaluadas en los dos grupos mediante la prueba $U$ de MannWhitney.

Sin embargo, ninguno de los análisis resultó significativo $\left(p_{\mathrm{s}}=0,15,0,24,0,09,0,33\right.$ y 0,75 , respectivamente, para bienestar emocional, bienestar físico, bienestar material, desarrollo personal y relaciones interpersonales).

También se llevaron a cabo correlaciones bivariadas no paramétricas, mediante la prueba $\rho$ de Spearman, entre las puntuaciones obtenidas en las distintas dimensiones. La única que resultó significativa para toda la muestra fue la establecida entre la dimensión de bienestar emocional y la de relaciones interpersonales $(r=0,48 ; p<0,05)$.

En el caso de los adolescentes, además resultaron significativas las correlaciones que se establecieron entre bienestar físico y autodeterminación $(r=0,75 ; p<0,01)$ y entre bienestar material e integración en la comunidad $(r=0,66 ; p<0,05)$.

Por último, se correlacionó la edad cronológica y el CIT con la puntuación obtenida en cada una de las dimensiones, y resultó significativa únicamente para el grupo de adolescentes entre el CIT y el área de integración en la comunidad $(r=0,81 ; p<0,01)$.

\section{Discusión}

A pesar del carácter preliminar de los resultados obtenidos en el presente estudio y de las limitaciones que supone el reducido tamaño de la muestra, es posible realizar algunas observaciones que resultan de interés para futuras investigaciones.

En primer lugar, parece que el grupo de los niños tiene una buena percepción sobre su calidad de vida. No se observa que ninguna de las dimensiones ten- 
ga una especial relevancia para la percepción final que los niños expresan en relación con ella.

No obstante, en el grupo de los adolescentes se encuentran diferencias entre las puntuaciones obtenidas en la dimensión de autodeterminación y el resto de las áreas, exceptuando la que hace referencia al bienestar material. Parece, por lo tanto, que los adolescentes con TEA sin discapacidad intelectual perciben que disponen de escasas oportunidades para tomar decisiones por sí mismos, y que las decisiones que adoptan tienen una repercusión limitada en sus vidas.

Aunque sería necesario realizar un análisis pormenorizado de las respuestas facilitadas en la dimensión de bienestar material, es posible aventurar que la ausencia de diferencias entre ambas dimensiones podría relacionarse con los elementos de esta última que hacen referencia al disfrute de las propias pertenencias y a tener oportunidades para administrar el propio dinero de cada uno.

Si esto fuera así, se aportaría un dato complementario que apoyaría la baja percepción de control y las escasas opciones para tomar decisiones que manifiestan los adolescentes, en este caso en relación con la administración de sus ingresos.

No obstante, sería interesante contrastar esta información con la percepción que las familias tienen en relación con la autonomía personal y las habilidades de autodirección de sus hijos adolescentes [17], así como con datos de evaluaciones objetivas sobre estas competencias [15]. Esta información resultaría relevante para establecer si las dificultades para tomar decisiones se pueden atribuir a problemas reales de autonomía personal e independencia que presenten los adolescentes o, por el contrario, son el reflejo de una excesiva preocupación y limitación de estas competencias por parte de sus familias.

En algunos estudios se pone de manifiesto la existencia de diferencias significativas entre las percepciones que los adolescentes con TEA sin discapacidad intelectual tienen sobre sus necesidades y las que reflejan sus familiares [21], que son más negativas y extremas en el segundo caso.

Hasta qué punto estas diferencias se pueden atribuir a las dificultades que el adolescente, debido a las propias características del autismo, puede tener para realizar una valoración realista de sus competencias interpersonales y adaptativas o, por el contrario, responden a una excesiva preocupación por parte de sus familiares, supone una cuestión que merece la pena explorar debido a las implicaciones que este aspecto tiene para la intervención clínica.

En el primer caso sería fundamental priorizar el desarrollo de las habilidades de autoconciencia y de autonomía personal de los adolescentes con TEA sin discapacidad intelectual, de manera que se promuevan sus competencias personales y adquieran un mayor control sobre sus vidas [30].

Sin embargo, en el segundo caso sería necesario trabajar de manera específica con las familias para que favorezcan el desarrollo personal de sus hijos sin caer en la sobreprotección, que limitaría su autonomía e independencia.

Asimismo, y de cara al futuro desarrollo de esta línea de investigación, será necesario explorar hasta qué punto pueden influir en los resultados la existencia de trastornos comórbidos (trastorno por déficit de atención/hiperactividad, trastornos del estado de ánimo, trastornos de ansiedad...), así como las manifestaciones diferenciales que pueden presentarse en las distintas etapas vitales. Debido al carácter exploratorio y preliminar de este estudio, estos aspectos no se han considerado de manera específica, pero sin duda tienen una relevancia fundamental en la percepción subjetiva que los niños y adolescentes con TEA pueden tener sobre su calidad de vida y también sobre el abordaje clínico de las intervenciones que puedan requerir [31-33].

Por otro lado, y aunque según los resultados obtenidos no es posible establecer conclusiones sólidas sobre la valoración subjetiva que los niños y adolescentes con TEA sin discapacidad intelectual hacen de su calidad de vida, resulta necesario reflexionar sobre la influencia que los cuestionarios empleados en la recogida de la información pueden tener en los resultados obtenidos [21].

Sería necesario observar si se han producido incidencias repetidas en la interpretación y en las respuestas proporcionadas a algunos ítems, especialmente en los que implican algunas de las competencias cognitivas más alteradas en los TEA sin discapacidad intelectual (por ejemplo, valoraciones emocionales, interpretar intenciones de los demás, comprender el lenguaje figurado o no literal de los enunciados, etc.).

Si se detectaran este tipo de problemas, se pondría de manifiesto la necesidad de desarrollar instrumentos y metodologías apropiadas para la evaluación de la percepción subjetiva y de la satisfacción personal en este tipo de trastornos. Sus propias características afectan a las competencias cognitivas requeridas para valorar la experiencia personal, por lo que los instrumentos actuales pueden no resultar apropiados si no se realizan adaptaciones en ellos. Además, y planteándose un objetivo más ambicioso, podría resultar de interés el análisis de las repercusiones que la intervención clínica especializada podría tener en el desarrollo de dichas competen- 
cias cognitivas, y sus implicaciones para la mejora de la calidad de vida subjetiva de las personas con TEA sin discapacidad intelectual.

En cualquier caso, las limitaciones de este estudio repercuten en las conclusiones que se puedan establecer y sólo permiten señalar áreas de interés para el fututo.

Debe considerarse que existen pocas diferencias en las valoraciones realizadas por los distintos individuos en relación con su calidad de vida. Es posible que el procedimiento para acceder a estos participantes, a través de servicios clínicos y educativos especializados en los apoyos a este colectivo, haya influido en esto, porque la mayor parte de las necesidades que estas personas manifiestan y que repercuten en su calidad de vida se cubre desde dichos servicios. Sería recomendable incrementar el tamaño de la muestra y también su representatividad, aunque ambos aspectos son complejos en este caso, al tratarse de un colectivo reducido y accesible principalmente a través de recursos especializados.

Asimismo, sería necesario incorporar otras medidas, incluyendo valoraciones objetivas sobre competencias personales, que permitieran complementar la información de los autoinformes con datos objetivos y también con las percepciones que tienen los familiares o los compañeros de la misma edad. Esto permitiría contrastar la información proporcionada por el niño o el adolescente y valorar si los resultados reflejan realmente (o no) su percepción subjetiva [34].

\section{Bibliografía}

1. Barthélémy C, Fuentes J, Howlin P, Van der Gaag R. Persons with autism spectrum disorders. Identification, understanding, intervention. Brussels: Autismo Europa; 2008.

2. American Psychiatric Association. Diagnostic and statistical manual of mental disorders, fifth edition (DSM-5). Washington DC: APA; 2013

3. Artigas-Pallarés J, Paula-Pérez I. Asignaturas pendientes del DSM-5. Rev Neurol 2015; 60 (Supl 1): S95-101.

4. Zamora M, Arnaiz J. Detección, diagnóstico y evaluación en Asperger. En Merino M, Zamora M, eds. Todo sobre el Asperger. Zaragoza; Altaria; 2014. p. 77-112.

5. Matson JL, Neel-Schwalm M. Comorbid psychophatology with autism spectrum disorders in children. Res Dev Disabil 2005; 28: 341-52.

6. Paula-Pérez I. Concurrencia entre ansiedad y autismo. Las hipótesis del error social y de la carga alostática. Rev Neurol 2013; 56 (Supl 1): S45-59.

7. Portway S, Johnson B. Do you know I have Asperger's syndrome? Risks of a non-obvious disability Health Risk Soc 2005; 7: 73-83.

8. Martín P. El síndrome de Asperger. ¿Excentricidad o discapacidad social? Madrid: Alianza; 2004.

9. Belinchón M, Hernández JM, Sotillo, M. Personas con síndrome de Asperger. Funcionamiento, detección y necesidades. Madrid: Fundación ONCE; 2008.

10. Schalock RL, Verdugo MA. Calidad de vida. Manual para profesionales de la educación, salud y los servicios sociales. Madrid: Alianza; 2003.
11. Schalock RL, Verdugo MA. El concepto de calidad de vida en los servicios y apoyos para personas con discapacidad intelectual. Siglo Cero 2007; 38: 21-36.

12. Schalock RL, Verdugo MA. El cambio en las organizaciones de discapacidad. Madrid: Alianza; 2012.

13. Verdugo MA, Gómez LE, Arias, B, Santamaría M, Clavero D, Tamarit J. Escala INICO-FEAPS: evaluación integral de la calidad de vida de personas con discapacidad intelectual o del desarrollo. Salamanca: INICO; 2013.

14. Schippers A. Quality of life in disability studies. Medische Antropologie 2010; 22: 277-87.

15. Burgess A, Gutstein S. Quality of life for people with autism Raising the standard for evaluating successful outcomes. Child Adolesc Ment Health 2007; 12: 80-6.

16. Renty J, Roeyers H. Quality of life in high functioning adults with autism spectrum disorders. The predictive value of disability and support characteristics. Autism 2006; 10: 511-24.

17. Sheldick R, Neger E, Shipman D, Perrin E. Quality of life of adolescents with autism spectrum disorders: concordance among adolescents' self- reports, parents reports, and parents proxy reports. Qual Life Res 2012; 21: 53-7.

18. Koning CI. Youth with autism spectrum disorders. Self- and proxy-reported quality of life and adaptive functioning. Focus Autism Other Dev Disabil 2015; 30: 57-64.

19. Van Heijst BF, Geurts HM. Quality of life in autism across the lifespan: a meta-analysis. Autism 2015; 19: 158-67.

20. Saldaña D, Álvarez RM, Lobatón S, López, AM, Moreno, M, Rojano, M. Objective and subjective quality of life in adults with autism spectrum disorders in southern Spain. Autism 2009; 13: 303-16.

21. Shipman D, Sheldrick C, Perrin E. Quality of life in adolescents with autism spectrum disorders: reliability and validity of self reports. J Dev Behav Pediatr 2011; 32: 85-9.

22. Cuesta JL. Trastornos del espectro autista y calidad de vida: guía de indicadores para evaluar organizaciones y servicios. Madrid: La Muralla; 2009.

23. Cuesta JL, Hortiguela V, coords. Senda hacia la participación. Calidad de vida en las personas con trastornos del espectro autista y sus familias. Burgos: Autismo Burgos; 2007.

24. Gerber F, Baud MA, Giroud M, Galli-Carminati G. Quality of life of adults with pervasive developmental disorders and intellectual disabilities. J Autism Dev Disord 2008; 38: 1654-65.

25. Plimley LA. A review of quality of life issues and people with autism spectrum disorders. Br J Learn Disabil 2007; 35: 205-13.

26. Billestedt E, Gillberg IC, Gillberg C. Aspects of quality of life in adults diagnosed with autism in childhood. Autism 2011; 15: 7-20.

27. Lord C, Risi S, Lambrecht L, Cook EH, Leventhal B, Dilavore PC The autism diagnostic observation schedule-generic: a standard measure of social and communication deficits associated with the autism spectrum. J Autism Dev Disord 2000; 30: 205-23.

28. Sabeh E, Verdugo MA, Prieto M, Contini de González C. Cuestionarios de evaluación de calidad de vida en la infancia (CVI-CVIP). Madrid: CEPE; 2009.

29. Gómez-Vela M, Verdugo MA. Cuestionario de evaluación de la calidad de vida de alumnos adolescentes (CCVA). Madrid: CEPE; 2009.

30. Wehmeyer M, Schalock R. Self-determination and quality of life: implications for special education services and support. Focus on Exceptional Children 2001; 33: 1-16.

31. Van Steensel FJ, Bögels SM. CBT for anxiety disorders in children with and without autism spectrum disorders. J Consult Clin Psychol 2015; 83: 512-23.

32. Van Steensel FJ, Bögels SM. Dirksen CD. Anxiety and quality of life: clinically anxious children with and without autism spectrum disorders compared. J Consult Clin Psychol 2012; 41: 731-8.

33. De la Iglesia $M$, Olivar S. Risk factors for depression in children and adolescents with high functioning autism spectrum disorders. Sci World J 2015; 2015: 127853.

34. Loveridge J, Meyer R. Children and young people with disabilities. In Loveridge J, ed. Involving children and young people in research in educational settings. Wellington, New Zealand: Victoria University of Wellington; 2010. p. 137-58. 


\section{Quality of life in children and adolescents with autism spectrum disorder without intellectual disability}

Introduction. The concept of quality of life is of great importance for interventions directed at people living with disabilities. It is undeniable that the achievement of a satisfactory quality of life should be the objective of clinical interventions that are directed at the group of people with autism spectrum disorder (ASD) and should be a key element in the design of the treatments that are needed to improve the prognosis of future development. Nevertheless, knowledge of both the objective and the subjective conditions that predict greater quality of life satisfaction in people with ASD is still very limited.

Aim. To explore the perception of the quality of life experienced by a group of children and adolescents with ASD without intellectual disability.

Patients and methods. A sample of 12 children and 11 adolescents with ASD without intellectual disability. In accordance with their age, they were administered the corresponding quality of life questionnaires.

Results. Both groups reported positively on their quality of life, without any differences in the basic dimensions. Nevertheless, in the case of adolescents, very low scores were obtained in the area of self-determination in comparison with their scores in other areas.

Conclusions. The discussion centers on the identification of the most relevant dimensions for quality of life, the implications for intervention, and the need to adapt the methodologies in use for the subjective evaluation of those factors, due to the characteristics of ASD that can affect the capabilities necessary for the evaluation of personal experience, and may require the adaptation of the procedures that are employed.

Key words. Asperger syndrome. Autism. Autism spectrum disorders. Diagnostic self-evaluation. Personal satisfaction. Qualitative evaluation. Quality of life. 